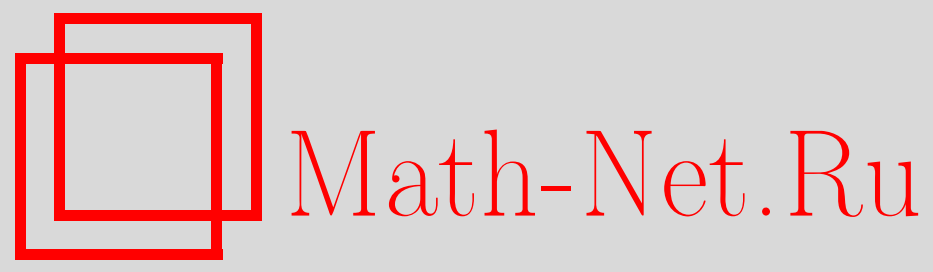

В. А. Емеличев, А. В. Пашкевич, Об условиях эффективности решения многокритериальной дискретной задачи, Дискрет. матем., 2002, том 14, выпуск 1, 17-29

DOI: https://doi.org/10.4213/dm225

Использование Общероссийского математического портала Math-Net.Ru подразумевает, что вы прочитали и согласны с пользовательским соглашением http://www . mathnet.ru/rus/agreement

Параметры загрузки:

IP : 54.157 .27 .8

26 апреля 2023 г., 13:39:46 
УДК 519.6

\title{
Об условиях эффективности решения многокритериальной дискретной задачи
}

\author{
(C) 2002 г. . А. Емеличев, А. В. Пашкевич
}

Для широкого класса многокритериальных (векторных) задач оптимизации с конечным множеством векторных оценок на основе аддитивного метода агрегирования специальных монотонных функций от частных критериев получены необходимые и достаточные условия эффективности (оптимальности по Парето) решения.

Работа выполнена в рамках Государственной программы фундаментальных исследований Республики Беларусь «Алгоритм».

\section{1. Введение}

Необходимые и достаточные условия того, чтобы данное решение многокритериальной задачи было эффективным (оптимальным по Парето), обычно формулируются в зависимости от свойств частных критериев и структуры множества решений (см., например, $[1,2])$. Знание таких условий служит основой для разработки методов нахождения эффективных решений, а также способов проверки эффективности выбранного решения.

Целью настоящей статьи является обобщение некоторых результатов работ [3, 4], где для многокритериальных дискретных задач (задач с конечным множеством векторных оценок) в терминах линейной свертки видоизмененных частных критериев и в терминах суммы положительных степеней частных критериев найдены необходимые и достаточные условия эффективности решения. Здесь эти условия формулируются в терминах суммы специальных монотонных функций от частных критериев. Получен ряд следствий, позволяющих искомое множество Парето представить в виде множества решений параметрической скалярной дискретной задачи оптимизации.

\section{2. Определения}

Пусть на множестве (допустимых) решений $X$ произвольной природы задана векторфункция

$$
f=\left(f_{1}, f_{2}, \ldots, f_{n}\right): X \rightarrow \mathbf{R}^{n}, \quad n \geqslant 2,
$$

компоненты которой (частные критерии) без потери общности будем считать минимизируемыми:

$$
f_{i}(x) \rightarrow \min _{x \in X}, \quad i \in N_{n}=\{1,2, \ldots, n\}
$$


Определение 1. Следуя $[3,4], n$-критериальной дискретной задачей $(n-\mathrm{K} Д 3)$ назовем задачу нахождения множества эффективных решений (множества Парето)

$$
P(X, f)=\{x \in X: \pi(x, f)=\varnothing\},
$$

где

$$
\pi(x, f)=\left\{x^{\prime} \in X: f(x) \geqslant f\left(x^{\prime}\right), f(x) \neq f\left(x^{\prime}\right)\right\}
$$

при условии конечности образа множества $X$ в критериальном пространстве, то есть конечности множества векторных оценок

$$
f(X)=\left\{y \in \mathbf{R}^{n}: y=f(x), x \in X\right\} .
$$

Эту задачу будем обозначать через $(X, f)$, а через $f_{i}(X)$ - проекцию множества $f(X)$ на ось $i$ :

$$
f_{i}(X)=\left\{y \in \mathbf{R}: y=f_{i}(x), x \in X\right\}, \quad i \in N_{n} .
$$

Без потери общности можно в дальнейшем предполагать, что задача $(X, f)$ такова, что

$$
\forall i \in N_{n} \quad \exists x, x^{\prime} \in X \quad\left(f_{i}(x) \neq f_{i}\left(x^{\prime}\right)\right) .
$$

В самом деле, пусть $J$ - множество тех индексов из $N_{n}$, для которых условие (1) не выполняется. Легко видеть, что в случае, когда $J \neq N_{n}$, можно перейти к эквивалентной задаче (то есть к задаче с тем же множеством Парето) путем исключения тех критериев, которые занумерованы числами из $J$. Очевидно, что в новой задаче условие (1) будет выполняться. Если $J=N_{n}$, то каждое решение $x n$-КдЗ $(X, f)$ эффективно.

Ясно, что в случае, когда выполняется свойство (1), справедливо неравенство $|f(X)| \geqslant 2$, которое в дальнейшем будем учитывать.

Для всякого натурального числа $m$ будем использовать обозначения

$$
\begin{aligned}
\mathbf{R}_{+}^{m} & =\left\{z \in \mathbf{R}^{m}: z_{i} \geqslant 0, i \in N_{m}\right\} \\
\mathbf{R}_{++}^{m} & =\left\{z \in \mathbf{R}^{m}: z_{i}>0, i \in N_{m}\right\}, \\
\mathbf{R}_{--}^{m} & =\left\{z \in \mathbf{R}^{m}: z_{i}<0, i \in N_{m}\right\} .
\end{aligned}
$$

Определение 2. Пусть $U \subset \mathbf{R}^{n},|U| \geqslant 2, i \in N_{n}, a \geqslant 0, \hat{v}>a$,

$$
U_{i}=\left\{z \in \mathbf{R}: z=u_{i}, u=\left(u_{1}, \ldots, u_{i}, \ldots, u_{n}\right) \in U\right\} .
$$

Будем говорить, что вектор-функция

$$
\xi(u, v)=\left(\xi_{1}\left(u_{1}, v\right), \xi_{2}\left(u_{2}, v\right), \ldots, \xi_{n}\left(u_{n}, v\right)\right)
$$

принадлежит классу $\Xi(U, a, \hat{v})$ и писать $\xi \in \Xi(U, a, \hat{v})$, если выполняются следующие условия:

$$
\begin{gathered}
\forall i \in N_{n} \quad\left(\xi_{i}: U_{i} \times(a, \infty) \rightarrow \mathbf{R}_{+}\right), \\
\forall i \in N_{n} \quad \forall v>a \quad \forall u, u^{\prime} \in U \quad\left(u_{i}>u_{i}^{\prime} \Rightarrow \xi_{i}\left(u_{i}, v\right)>\xi_{i}\left(u_{i}^{\prime}, v\right)\right), \\
\forall i \in N_{n} \quad \forall v \geqslant \hat{v} \quad \forall u, u^{\prime} \in U \quad\left(u_{i}>u_{i}^{\prime} \Rightarrow \xi_{i}\left(u_{i}, v\right) \geqslant n \xi_{i}\left(u_{i}^{\prime}, v\right)\right) .
\end{gathered}
$$

Существование таких вектор-функций очевидно (см. ниже п. 4). 


\section{3. Теоремы}

Теорема 1 (достаточное условие). Пусть вектор-функция

$$
\xi(u, v)=\left(\xi_{1}\left(u_{1}, v\right), \xi_{2}\left(u_{2}, v\right), \ldots, \xi_{n}\left(u_{n}, v\right)\right),
$$

каждая $i$-я компонента которой определена на множестве $f_{i}(X) \times(a, \infty), a \geqslant 0$, удовлетворяет условию (3). Тогда при любом иисле $v>$ а решение $x^{*} n-K Д З(X, f)$, для которого верно равенство

$$
\sum_{i=1}^{n} \xi_{i}\left(f_{i}\left(x^{*}\right), v\right)=\min \left\{\sum_{i=1}^{n} \xi_{i}\left(f_{i}(x), v\right): x \in X\right\},
$$

является эффективным.

Доказательство. Предположим противное, то есть решение $x^{*} \in X$, удовлетворяя равенству (5), не является эффективным. Тогда в силу внешней устойчивости $n$-КДЗ $(X, f)[1]$ существует такое решение $x^{0} \in P(X, f)$, что

$$
f\left(x^{*}\right) \geqslant f\left(x^{0}\right), \quad f\left(x^{*}\right) \neq f\left(x^{0}\right) .
$$

Отсюда, учитывая условие (3), находим, что при любом $v>a$ справедливы неравенства

$$
\xi_{i}\left(f_{i}\left(x^{*}\right), v\right) \geqslant \xi_{i}\left(f_{i}\left(x^{0}\right), v\right), \quad i \in N_{n},
$$

причем хотя бы одно из них строгое. Поэтому при любом $v>a$

$$
\sum_{i=1}^{n} \xi_{i}\left(f_{i}\left(x^{*}\right), v\right)>\sum_{i=1}^{n} \xi_{i}\left(f_{i}\left(x^{0}\right), v\right)
$$

что противоречит равенству (5).

Теорема 1 доказана.

Теорема 2 (необходимое условие). Пусть $\xi \in \Xi(f(X), a, \hat{v})$ и решение $x^{*} \in P(X, f)$, причем для любого числа $v>а$ выполняются равенства

$$
\xi_{1}\left(f_{1}\left(x^{*}\right), v\right)=\xi_{2}\left(f_{2}\left(x^{*}\right), v\right)=\cdots=\xi_{n}\left(f_{n}\left(x^{*}\right), v\right) .
$$

Тогда при любом $v \geqslant \hat{v}$ верно равенство

$$
n \xi_{1}\left(f_{1}\left(x^{*}\right), v\right)=\min \left\{\sum_{i=1}^{n} \xi_{i}\left(f_{i}(x), v\right): x \in X\right\} .
$$

Доказательство. Пусть $x^{*} \in P(X, f)$. Разобьем множество $X$ на два непересекающихся подмножества

$$
\begin{aligned}
& X_{1}=\left\{x \in X:\left[x^{*}, x\right]_{-} \cup\left[x^{*}, x\right]_{0}=N_{n}\right\} \\
& X_{2}=\left\{x \in X:\left[x^{*}, x\right]_{+} \neq \varnothing\right\}
\end{aligned}
$$


где

$$
\begin{gathered}
{\left[x^{*}, x\right]_{+}=\left\{i \in N_{n}: f_{i}\left(x^{*}\right)>f_{i}(x)\right\},} \\
{\left[x^{*}, x\right]_{-}=\left\{i \in N_{n}: f_{i}\left(x^{*}\right)<f_{i}(x)\right\},} \\
{\left[x^{*}, x\right]_{0}=\left\{i \in N_{n}: f_{i}\left(x^{*}\right)=f_{i}(x)\right\} .}
\end{gathered}
$$

Пусть $x \in X_{1}$. Тогда при любом индексе $i \in N_{n}$ очевидно неравенство $f_{i}\left(x^{*}\right) \leqslant$ $f_{i}(x)$. Поэтому для всякого $v>a$ согласно (3) верны неравенства

$$
\xi_{i}\left(f_{i}\left(x^{*}\right), v\right) \leqslant \xi_{i}\left(f_{i}(x), v\right), \quad i \in N_{n},
$$

а следовательно, с учетом (6), и неравенство

$$
n \xi_{1}\left(f_{1}\left(x^{*}\right), v\right) \leqslant \sum_{i=1}^{n} \xi_{i}\left(f_{i}(x), v\right) .
$$

Далее покажем, что это неравенство справедливо для любого решения $x \in X_{2}$ при всяком $v \geqslant \hat{v}$.

Пусть $x \in X_{2}$. Тогда $\left[x^{*}, x\right]_{+} \neq \varnothing$. Так как $x^{*} \in P(X, f)$, то $\left[x^{*}, x\right]_{-}=\left[x, x^{*}\right]_{+} \neq \varnothing$. Зафиксируем произвольное число $v \geqslant \hat{v}$. Пусть далее

$$
\begin{gathered}
k \in \arg \max \left\{\xi_{i}\left(f_{i}\left(x^{*}\right), v\right)-\xi_{i}\left(f_{i}(x), v\right): i \in\left[x^{*}, x\right]_{+}\right\}, \\
l \in \arg \min \left\{\xi_{j}\left(f_{j}(x), v\right)-\xi_{j}\left(f_{j}\left(x^{*}\right), v\right): j \in\left[x, x^{*}\right]_{+}\right\} .
\end{gathered}
$$

Тогда, учитывая неравенства

$$
f_{l}(x)>f_{l}\left(x^{*}\right), \quad v \geqslant \hat{v},
$$

и применяя условие (4), убеждаемся, что верно неравенство

$$
\xi_{l}\left(f_{l}(x), v\right) \geqslant n \xi_{l}\left(f_{l}\left(x^{*}\right), v\right) .
$$

Отсюда, согласно (2) и (6), выводим, что

$$
\begin{aligned}
\xi_{l}\left(f_{l}(x), v\right)-\xi_{l}\left(f_{l}\left(x^{*}\right), v\right) & \geqslant(n-1) \xi_{l}\left(f_{l}\left(x^{*}\right), v\right) \\
& =(n-1) \xi_{k}\left(f_{k}\left(x^{*}\right), v\right) \\
& \geqslant(n-1)\left(\xi_{k}\left(f_{k}\left(x^{*}\right), v\right)-\xi_{k}\left(f_{k}\left(x^{\prime}\right), v\right)\right) .
\end{aligned}
$$

Поэтому для всякого решения $x \in X_{2}$ и всякого $v \geqslant \hat{v}$ верно неравенство (8), поскольку

$$
\begin{aligned}
& n \xi_{1}\left(f_{1}\left(x^{*}\right), v\right)-\sum_{i=1}^{n} \xi_{i}\left(f_{i}(x), v\right)=\sum_{i=1}^{n} \xi_{i}\left(f_{i}\left(x^{*}\right), v\right)-\sum_{i=1}^{n} \xi_{i}\left(f_{i}(x), v\right) \\
& =\sum_{i \in\left[x^{*}, x\right]_{+}}\left(\xi_{i}\left(f_{i}\left(x^{*}\right), v\right)-\xi_{i}\left(f_{i}(x), v\right)\right)-\sum_{j \in\left[x, x^{*}\right]_{+}}\left(\xi_{j}\left(f_{j}(x), v\right)-\xi_{j}\left(f_{j}\left(x^{*}\right), v\right)\right) \\
& \leqslant(n-1)\left(\xi_{k}\left(f_{k}\left(x^{*}\right), v\right)-\xi_{k}\left(f_{k}(x), v\right)\right)-\left(\xi_{l}\left(f_{l}(x), v\right)-\xi_{l}\left(f_{l}\left(x^{*}\right), v\right)\right) \leqslant 0 .
\end{aligned}
$$

Итак, при любом $v \geqslant \hat{v}$ неравенство (8) выполняется для всякого решения $x \in X$, то есть, верно равенство (7).

Теорема 2 доказана.

Подбирая вектор-функцию $\xi(u, v)$ подходящего вида, будем получать соответствующие конкретизации теорем 1 и 2 , которые сформулируем в виде следствий. Для доказательства этих следствий нам понадобится ряд лемм. 


\section{4. Леммы}

Всюду в этом пункте $n \geqslant 2-$ целое число.

Лемма 1. Пустъ $U \subset \mathbf{R}^{n}, 2 \leqslant|U|<\infty, t \in \mathbf{R}_{++}^{n}$. Тогда вектор-функция $\xi(u, v) c$ компонентами

$$
\xi_{i}\left(u_{i}, v\right)=v^{u_{i} / t_{i}}, \quad i \in N_{n},
$$

принадлежит классу $\Xi(U, 1, \hat{v})$, где

$$
\begin{aligned}
& \hat{v}=n^{\tau / \delta} \\
& \tau=\max \left\{t_{i}: i \in N_{n}\right\}, \\
& \delta=\min \left\{u_{i}-u_{i}^{\prime}>0: u, u^{\prime} \in U, i \in N_{n}\right\} .
\end{aligned}
$$

Доказателъство. Прежде всего заметим, что существование числа $\delta$ гарантируется неравенствами $2 \leqslant|U|<\infty$.

Очевидно, что вектор-функция $\xi(u, v)$ удовлетворяет условиям (2) и (3).

В выполнимости условия (4) легко убедиться, поскольку для любых $v \geqslant \hat{v}$, $u, u^{\prime} \in U$ и $i \in N_{n}$ таких, что $u_{i}>u_{i}^{\prime}$, с учетом условий леммы 1 имеем

$$
\frac{\xi_{i}\left(u_{i}, v\right)}{\xi_{i}\left(u_{i}^{\prime}, v\right)}=v^{\left(u_{i}-u_{i}^{\prime}\right) / t_{i}} \geqslant \hat{v}^{\left(u_{i}-u_{i}^{\prime}\right) / t_{i}}=n^{\tau\left(u_{i}-u_{i}^{\prime}\right) /\left(\delta t_{i}\right)} \geqslant n^{\left(u_{i}-u_{i}^{\prime}\right) / \delta} \geqslant n .
$$

Лемма 1 доказана.

Лемма 2. Пусть $U \subset \mathbf{R}_{+}^{n}, 2 \leqslant|U|<\infty, t \in \mathbf{R}_{++}^{n}$. Тогда вектор-функция $\xi(u, v) c$ компонентами

$$
\xi_{i}\left(u_{i}, v\right)=u_{i}^{v t_{i}}, \quad i \in N_{n},
$$

принадлежит классу $\Xi(U, 0, \hat{v})$, где

$$
\begin{aligned}
\hat{v} & = \begin{cases}\log n /(\tau \log \Delta), & \text { если число } \Delta \text { существует, } \\
1 & \text { в противном случае }\end{cases} \\
\tau & =\min \left\{t_{i}: i \in N_{n}\right\}, \\
\Delta & =\min \left\{u_{i} / u_{j}^{\prime}>1: u, u^{\prime} \in U, i, j \in N_{n}\right\}
\end{aligned}
$$

Доказателъство. Легко проверить, что вектор-функция $\xi(u, v)$ удовлетворяет условиям (2) и (3).

Докажем выполнимость условия (4). Пусть число $\Delta$ существует. С учетом включения $U \subset \mathbf{R}_{+}^{n}$, рассмотрим два возможных случая.

Пусть $u_{i}>u_{i}^{\prime}>0$. Тогда при любом $v \geqslant \hat{v}$

$$
\begin{aligned}
\frac{\xi_{i}\left(u_{i}, v\right)}{\xi_{i}\left(u_{i}^{\prime}, v\right)} & =\left(\frac{u_{i}}{u_{i}^{\prime}}\right)^{v t_{i}} \geqslant\left(\frac{u_{i}}{u_{i}^{\prime}}\right)^{\hat{v} t_{i}}=\left(\frac{u_{i}}{u_{i}^{\prime}}\right)^{t_{i} \log n /(\tau \log \Delta)} \\
& \geqslant\left(\frac{u_{i}}{u_{i}^{\prime}}\right)^{\log n / \log \Delta} \geqslant \Delta^{\log n / \log \Delta}=n
\end{aligned}
$$

то есть условие (4) выполняется.

Пусть теперь $u_{i}>u_{i}^{\prime}=0$. Тогда при любом $v \geqslant \hat{v}$

$$
\xi_{i}\left(u_{i}, v\right)=u_{i}^{v t_{i}}>0=n \xi_{i}\left(u_{i}^{\prime}, v\right),
$$


то есть условие (4) выполняется и в этом случае.

Если же числа $\Delta$ не существует, то, учитывая неравенство $|U| \geqslant 2$ и формулу, задающую $\Delta$, убеждаемся, что при любых $i \in N_{n}$ и $u \in U$ справедливо включение $u_{i} \in\{0, b\}$, где $b>0$. Тогда из неравенства $u_{i}>u_{i}^{\prime}$ следует, что $u_{i}=b$ и $u_{i}^{\prime}=0$. Поэтому, принимая во внимание неравенство $v \geqslant \hat{v}=1$, получаем, что

$$
\xi_{i}\left(u_{i}, v\right)=b^{v t_{i}}>0=n \xi_{i}\left(u_{i}^{\prime}, v\right) .
$$

Следовательно, импликация (4) имеет место и в этом случае.

Итак, $\xi \in \Xi(U, 0, \hat{v})$.

Лемма 2 доказана.

Очевидно следующее утверждекие.

Лемма 3. Если $\xi \in \Xi(U, a, \hat{v}) u t \in \mathbf{R}_{++}^{n}$, то вектор-функция $\bar{\xi}(u, v)$ с компонентами

$$
\bar{\xi}_{i}\left(u_{i}, v\right)=t_{i} \xi\left(u_{i}, v\right), \quad i \in N_{n},
$$

принадлежит классу $\Xi(U, a, \hat{v})$.

Лемма 4. Пусть $\xi \in \Xi(U, a, \hat{v}) u u^{*}=\left(u_{1}^{*}, u_{2}^{*}, \ldots, u_{n}^{*}\right) \in U$ таковъ, ито при любъх $v>a u i \in N_{n}$ справедливо неравенство $\xi_{i}\left(u_{i}^{*}, v\right)>0$. Тогда вектор-функция $\bar{\xi}(u, v)$ с компонентами

$$
\bar{\xi}_{i}\left(u_{i}, v\right)=\frac{\xi_{i}\left(u_{i}, v\right)}{\xi_{i}\left(u_{i}^{*}, v\right)}, \quad i \in N_{n}
$$

принадлежит классу $\Xi(U, a, \hat{v})$.

Дожазателъство. Выполнимость условия

$$
\forall i \in N_{n} \quad\left(\bar{\xi}_{i}: U_{i} \times(a, \infty) \rightarrow \mathbf{R}_{+}\right)
$$

очевидна.

Так как $\xi \in \Xi(U, a, \hat{v})$, из условия (3), учитывая неравенство $\xi_{i}\left(u_{i}^{*}, v\right)>0$, справедливое при любых $v>a$ и $i \in N_{n}$, получаем, что

$$
\forall i \in N_{n} \quad \forall v>a \quad \forall u, u^{\prime} \in U \quad\left(u_{i}>u_{i}^{\prime} \Rightarrow \bar{\xi}_{i}\left(u_{i}, v\right)>\bar{\xi}_{i}\left(u_{i}^{\prime}, v\right)\right) .
$$

Поскольку $\xi \in \Xi(U, a, \hat{v})$, верна формула (4). Поэтому, принимая во внимание неравенство $\xi_{i}\left(u_{i}^{*}, v\right)>0$, справедливое при любых $v>a$ и $i \in N_{n}$, находим, что

$$
\forall i \in N_{n} \quad \forall v \geqslant \hat{v} \quad \forall u, u^{\prime} \in U \quad\left(u_{i}>u_{i}^{\prime} \Rightarrow \bar{\xi}_{i}\left(u_{i}, v\right) \geqslant n \bar{\xi}_{i}\left(u_{i}^{\prime}, v\right)\right) .
$$

Итак, $\bar{\xi} \in \Xi(U, a, \hat{v})$.

Лемма 4 доказана.

Как и прежде (см. определение 2 ) через $U_{i}$ будем обозначать проекцию множества $U$ на ось $i, i \in N_{n}$.

Лемма 5. Если $U \subset \mathbf{R}^{n}, 2 \leqslant|U|<\infty, \eta_{i}\left(u_{i}\right), i \in N_{n},-$ возрастающие функции

$$
\eta_{i}: U_{i} \rightarrow \mathbf{R}_{+}
$$


то вектор-функция $\xi(u, v)$ с компонентами

$$
\xi_{i}\left(u_{i}, v\right)=\eta_{i}^{v}\left(u_{i}\right), \quad i \in N_{n},
$$

принадлежит классу $\Xi(U, 0, \hat{v})$, где

$$
\begin{aligned}
\hat{v} & = \begin{cases}\log n / \log \Delta, & \text { если число } \Delta \text { существует, } \\
1 & \text { в противном случае },\end{cases} \\
\Delta & =\min \left\{\eta_{i}\left(u_{i}\right) / \eta_{j}\left(u_{j}^{\prime}\right)>1: u, u^{\prime} \in U, i, j \in N_{n}\right\} .
\end{aligned}
$$

Доказательство. Легко проверить, что в силу условий леммы 5 вектор-функция $\xi(u, v)$ удовлетворяет условиям (2) и (3).

Докажем теперь выполнимость условия (4). Пусть число $\Delta$ существует. Принимая во внимание неотрицательную определенность функций $\eta_{i}$, рассмотрим два возможных случая.

Пусть $u_{i}>u_{i}^{\prime}$ и $\eta_{i}\left(u_{i}^{\prime}\right)>0$. Тогда при всяком $v \geqslant \hat{v}$

$$
\frac{\xi_{i}\left(u_{i}, v\right)}{\xi_{i}\left(u_{i}^{\prime}, v\right)}=\left(\frac{\eta_{i}\left(u_{i}\right)}{\eta_{i}\left(u_{i}^{\prime}\right)}\right)^{v} \geqslant\left(\frac{\eta_{i}\left(u_{i}\right)}{\eta_{i}\left(u_{i}^{\prime}\right)}\right)^{\hat{v}}=\left(\frac{\eta_{i}\left(u_{i}\right)}{\eta_{i}\left(u_{i}^{\prime}\right)}\right)^{\log n / \log \Delta} \geqslant \Delta^{\log n / \log \Delta}=n .
$$

Пусть теперь $u_{i}>u_{i}^{\prime}$ и $\eta_{i}\left(u_{i}^{\prime}\right)=0$. Тогда в силу возрастания функции $\eta_{i}$ справедливо неравенство $\eta_{i}\left(u_{i}\right)>0$. Поэтому при любом $v \geqslant \hat{v}$

$$
\xi_{i}\left(u_{i}, v\right)=\eta_{i}^{v}\left(u_{i}\right)>0=n \xi_{i}\left(u_{i}^{\prime}, v\right) .
$$

Если же число $\Delta$ не существует, то, учитывая неравенство $|U| \geqslant 2$ и формулу, задающую $\Delta$, при всяком векторе $u \in U$ убеждаемся в справедливости включений $\eta_{i}\left(u_{i}\right) \in\{0, b\}, i \in N_{n}$, где $b>0$. Поэтому из неравенства $u_{i}>u_{i}^{\prime}$ с учетом возрастания функции $\eta_{i}$ следует, что $\eta_{i}\left(u_{i}\right)=b$ и $\eta_{i}\left(u_{i}^{\prime}\right)=0$. Отсюда получаем, что при любых $i \in N_{n}$ и $v \geqslant \hat{v}=1$ справедливы соотношения

$$
\xi_{i}\left(u_{i}, v\right)=\eta^{v}\left(u_{i}\right)=b^{v}>0=n \xi_{i}\left(u_{i}^{\prime}, v\right) .
$$

Следовательно, условие (4) имеет место и в этом случае.

Лемма 5 доказана.

\section{5. Следствия}

Во всех приведенных ниже следствиях речь идет о $n$-КДЗ $(X, f)$, для которой, как мы уже условились, $2 \leqslant|f(X)|<\infty, n \geqslant 2$.

Положим

$$
\mathbf{R}_{>1}^{n}=\left\{y \in \mathbf{R}^{n}: y_{i}>1, i \in N_{n}\right\}
$$

Следствие 1. При любом векторе $\varkappa \in \mathbf{R}_{>1}^{n}$ решение $x^{*} n-K Д 3(X, f)$, удовлетворяющее равенству

$$
\sum_{i=1}^{n} \varkappa_{i}^{f_{i}\left(x^{*}\right)}=\min \left\{\sum_{i=1}^{n} \varkappa_{i}^{f_{i}(x)}: x \in X\right\}
$$

является эфбективнъм.

Если для решения $x^{*} \in P(X, f)$ выполняется одно из двух условии $f\left(x^{*}\right) \in \mathbf{R}_{++}^{n}$ или $f\left(x^{*}\right) \in \mathbf{R}_{--}^{n}$, то найдется еектор $\varkappa \in \mathbf{R}_{>1}^{n}$, при котором справедливо равенство (12). 
Доказательство. Докажем первое утверждение. На основании леммы 1 векторфункция $\xi(u, v)$ с компонентами

$$
\xi_{i}\left(u_{i}, v\right)=v^{u_{i} / t_{i}}, \quad i \in N_{n},
$$

при любом $t \in \mathbf{R}_{++}^{n}$ принадлежит классу $\Xi(U, 1, \hat{v})$, где $\hat{v}$ задается формулой (9). Поэтому в силу теоремы 1 при любых $v>1, t_{i}>0, i \in N_{n}$, решение $x^{*}$, удовлетворяющее равенству

$$
\sum_{i=1}^{n} v^{f_{i}\left(x^{*}\right) / t_{i}}=\min \left\{\sum_{i=1}^{n} v^{f_{i}(x) / t_{i}}: x \in X\right\},
$$

является эффективным.

Для завершения доказательства первого утверждения достаточно убедиться, что для всякого вектора $\varkappa \in \mathbf{R}_{>1}^{n}$ найдется число $v>1$ и вектор $t \in \mathbf{R}_{++}^{n}$ такие, что

$$
\varkappa_{i}=v^{1 / t_{i}}, \quad i \in N_{n} .
$$

Очевидно, что для этого при любом фиксированном $v>1$ в качестве $t$ следует брать вектор с компонентами

$$
t_{i}=\frac{\log v}{\log \varkappa_{i}}, \quad i \in N_{n} .
$$

Докажем второе утверждение следствия. Пусть $x^{*} \in P(X, f)$. Определим компоненты вектор-функции $\xi(u, v)$ следующим образом:

$$
\xi_{i}\left(u_{i}, v\right)=v^{u_{i} /\left|f_{i}\left(x^{*}\right)\right|}, \quad i \in N_{n} .
$$

На основании леммы 1 вектор-функция $\xi \in \Xi(f(X), 1, \hat{v})$, где $\hat{v}$ определяется по формуле (9). Далее, учитывая условия следствия 1 , состоящее в том, что $\operatorname{sign} f_{i}\left(x^{*}\right)$ одинаков для всех $i \in N_{n}$, при всяком $v>1$

$$
\xi_{1}\left(f_{1}\left(x^{*}\right), v\right)=\xi_{2}\left(f_{2}\left(x^{*}\right), v\right)=\ldots=\xi_{n}\left(f_{n}\left(x^{*}\right), v\right)=v^{\operatorname{sign} f_{1}\left(x^{*}\right)} .
$$

Отсюда в силу теоремы 2 при всяком $v \geqslant \hat{v}$ получаем, что

$$
n v^{\operatorname{sign} f_{1}\left(x^{*}\right)}=\min \left\{\sum_{i=1}^{n} v^{f_{i}(x) /\left|f_{i}\left(x^{*}\right)\right|}: x \in X\right\} .
$$

Следовательно, в качестве компонент вектора $\varkappa \in \mathbf{R}_{>1}^{n}$ можно взять любые числа $\varkappa_{i}=v^{1 /\left|f_{i}\left(x^{*}\right)\right|}, i \in N_{n}$, при $v \geqslant \hat{v}$.

Следствие 1 доказано.

Введем обозначение

$$
H(X, f)=\bigcup_{\varkappa \in \mathbf{R}_{>1}^{n}} H(X, f, \varkappa)
$$

где

$$
H(X, f, \varkappa)=\arg \min \left\{\sum_{i=1}^{n} \varkappa_{i}^{f_{i}(x)}: x \in X\right\} .
$$

Тогда из следствия 1 легко выводим следующее утверждение. 
Следствие 2. Если выполняется одно из двух условий $f(X) \subset \mathbf{R}_{++}^{n}$ uли $f(X) \subset$ $\mathbf{R}_{--}^{n}$, mo $P(X, f)=H(X, f)$.

Следствие 3 ([4]). Если $f(X) \subset \mathbf{R}_{+}^{n}$, то справедливы следующие утверждения.

При любом векторе $\varkappa \in \mathbf{R}_{++}^{n}$ решение $x^{*} n$-КДЗ $(X, f)$, удовлетворяющее равенству

$$
\sum_{i=1}^{n} f_{i}^{\varkappa_{i}}\left(x^{*}\right)=\min \left\{\sum_{i=1}^{n} f_{i}^{\varkappa_{i}}(x): x \in X\right\}
$$

\section{является эффективным.}

Если для решения $x^{*} \in P(X, f)$ верно одно из двух условий $0<f_{i}\left(x^{*}\right)<1$ для всех $i \in N_{n}$ или $f_{i}\left(x^{*}\right)>1$ для всех $i \in N_{n}$, то найдется вектор $\varkappa \in \mathbf{R}_{++}^{n}$, при котором справедливо равенство (13).

Доказательство. Докажем первое утверждение. На основании леммы 2 векторфункция $\xi(u, v)$ с компонентами

$$
\xi_{i}\left(u_{i}, v\right)=u_{i}^{v t_{i}}, \quad i \in N_{n},
$$

при любом $t \in \mathbf{R}_{++}^{n}$ принадлежит классу $\Xi(U, 0, \hat{v})$, где $\hat{v}$ задается формулой (10). Поэтому в силу теоремы 1 при любых $v>0, t_{i}>0, i \in N_{n}$, решение $x^{*}$, удовлетворяющее равенству

$$
\sum_{i=1}^{n} f_{i}^{v t_{i}}\left(x^{*}\right)=\min \left\{\sum_{i=1}^{n} f_{i}^{v t_{i}}(x): x \in X\right\},
$$

является эффективным.

Для завершения доказательства первого утверждения достаточно убедиться, что для всякого вектора $\varkappa \in \mathbf{R}_{++}^{n}$ найдется число $v>1$ и вектор $t \in \mathbf{R}_{++}^{n}$ такие, что

$$
\varkappa_{i}=v t_{i}, \quad i \in N_{n} .
$$

Очевидно, что для этого при любом фиксированном числе $v>1$ в качестве $t$ следует брать вектор с компонентами

$$
t_{i}=\frac{\varkappa_{i}}{v}, \quad i \in N_{n}
$$

Докажем теперь второе утверждение. Пусть $x^{*} \in P(X, f)$. Введем вектор-функцию $\xi(u, v)$ с компонентами

$$
\xi_{i}\left(u_{i}, v\right)=\dot{u}_{i}^{v t_{i}}, \quad i \in N_{n},
$$

где

$$
t_{i}=\frac{\log f_{1}\left(x^{*}\right)}{\log f_{i}\left(x^{*}\right)}>0 .
$$

Тогда по лемме 2 вектор-функция $\xi \in \Xi(f(X), 0, \hat{v})$, где $\hat{v}$ определяется по формуле (10). Кроме того, в силу строения вектор-функции $\xi(u, v)$ для всякого $v>0$ очевидны равенства

$$
\xi_{1}\left(f_{1}\left(x^{*}\right), v\right)=\xi_{2}\left(f_{2}\left(x^{*}\right), v\right)=\ldots=\xi_{n}\left(f_{n}\left(x^{*}\right), v\right)=f_{1}^{v}\left(x^{*}\right)
$$


Поэтому на основании теоремы 2 при всяком $v \geqslant \hat{v}$ верно равенство

$$
n f_{1}^{v}\left(x^{*}\right)=\min \left\{\sum_{i=1}^{n} f_{i}^{v t_{i}}(x): x \in X\right\} .
$$

Следовательно, в качестве компонент вектора $\varkappa \in \mathbf{R}_{++}^{n}$ можно взять любые числа $\varkappa_{i}=v t_{i}, i \in N_{n}$, при $v \geqslant \hat{v}$.

Следствие 3 доказано.

Учитывая это следствие, легко сформулировать утверждение, аналогичное следствию 2 , что предоставляется читателю.

Введем обозначение

$$
\Lambda_{n}=\left\{\lambda \in \mathbf{R}_{++}^{n}: \sum_{i=1}^{n} \lambda_{i}=1\right\}
$$

Следствие 4. Если вектор-фунхция $\xi \in \Xi(f(X), a, \hat{v})$, то имеют место следующие утверждения.

При любом векторе $\lambda \in \Lambda_{n}$ и любом числе $v>a$ решение $x^{*} n$-KДЗ $(X, f)$, удовлетворяющее равенству

$$
\sum_{i=1}^{n} \lambda_{i} \xi_{i}\left(f_{i}\left(x^{*}\right), v\right)=\min \left\{\sum_{i=1}^{n} \lambda_{i} \xi_{i}\left(f_{i}(x), v\right): x \in X\right\}
$$

эффективно.

Если для решения $x^{*} \in P(X, f)$ неравенство $\xi_{i}\left(f_{i}\left(x^{*}\right), v\right)>0$ выполняется при любъх $v>a$ u $i \in N_{n}$, то при всяком $v \geqslant \hat{v}$ найдется вектор $\lambda \in \Lambda_{n}$, для которого верно равенство (14).

Доказательство. Докажем первое утверждение. Пусть $\lambda=\left(\lambda_{1}, \lambda_{2}, \ldots, \lambda_{n}\right) \in \Lambda_{n}$. На основании леммы 3 вектор-функция $\bar{\xi}(u, v)$ с компонентами

$$
\bar{\xi}_{i}\left(u_{i}, v\right)=\lambda_{i} \xi_{i}\left(f_{i}(x), v\right), \quad i \in N_{n},
$$

принадлежит классу $\Xi(f(X), a, \hat{v})$. Поэтому в силу теоремы 1 при любых $v>a$ и $\lambda \in \Lambda_{n}$ верно равенство (14).

Докажем второе утверждение. Пусть $x^{*} \in P(X, f)$. При любом $v>a$ зададим вектор

$$
\lambda=\lambda(v)=\left(\lambda_{1}, \lambda_{2}, \ldots, \lambda_{n}\right)
$$

где

$$
\begin{aligned}
\lambda_{i} & =\lambda_{i}(v)=\frac{L(v)}{\xi_{i}\left(f_{i}\left(x^{*}\right), v\right)}, \quad i \in N_{n}, \\
L(v) & =\left(\sum_{i=1}^{n} \frac{1}{\xi_{i}\left(f_{i}\left(x^{*}\right), v\right)}\right)^{-1} .
\end{aligned}
$$

Очевидно, что вектор $\lambda(v) \in \Lambda_{n}$ при любом $v>0$. 
Для всякого индекса $i \in N_{n}$ определим функцию

$$
\bar{\xi}_{i}\left(u_{i}, v\right)=\frac{\xi_{i}\left(u_{i}, v\right)}{\xi_{i}\left(f_{i}\left(x^{*}\right), v\right)}
$$

Нетрудно убедиться, что при любом $v>a$ выполняется равенство

$$
\sum_{i=1}^{n} \bar{\xi}_{i}\left(u_{i}, v\right)=\frac{1}{L(v)} \sum_{i=1}^{n} \lambda_{i} \xi_{i}\left(u_{i}, v\right)
$$

Согласно лемме 4 вектор-функция $\bar{\xi} \in \Xi(f(X), a, \hat{v})$, причем для всякого $v>a$ справедливы равенства

$$
\bar{\xi}_{1}\left(f_{1}\left(x^{*}\right), v\right)=\bar{\xi}_{2}\left(f_{2}\left(x^{*}\right), v\right)=\ldots=\bar{\xi}_{n}\left(f_{n}\left(x^{*}\right), v\right)=1
$$

Поэтому в силу теоремы 2 при любом $v \geqslant \hat{v}$

$$
\sum_{i=1}^{n} \bar{\xi}_{i}\left(f_{i}\left(x^{*}\right), v\right)=n=\min \left\{\sum_{i=1}^{n} \bar{\xi}_{i}\left(f_{i}(x), v\right): x \in X\right\} .
$$

Отсюда, учитывая (16) и неравенство $L(v)>0$, справедливое в силу условий следствия 4 при любом $v>a$, находим, что для всякого $v>a$ имеет место соотношение

$$
\sum_{i=1}^{n} \lambda_{i} \xi_{i}\left(f_{i}\left(x^{*}\right), v\right)=n L(v)=\min \left\{\sum_{i=1}^{n} \lambda_{i} \xi_{i}\left(f_{i}(x), v\right): x \in X\right\} .
$$

Таким образом, в качестве компонент вектора $\lambda \in \Lambda_{n}$ можно взять любые числа $\lambda_{i}=\lambda_{i}(v), i \in N_{n}$, задаваемые формулой (15) при $v \geqslant \hat{v}$.

Следствие 4 доказано.

Из следствия 4 получаем ряд сопутствующих результатов (см. ниже следствия 5-7).

Следствие 5 ([3]). IІри любом векторе $\lambda \in \Lambda_{n} u$ любом $v>1$ решение $x^{*} n-K Д 3$ $(X, f)$, удовлетворяющее равенству

$$
\sum_{i=1}^{n} \lambda_{i} v^{f_{i}\left(x^{*}\right)}=\min \left\{\sum_{i=1}^{n} \lambda_{i} v^{f_{i}(x)}: x \in X\right\}
$$

являетсл эффективным.

Если $x^{*} \in P(X, f)$, то существует такое число $\hat{v}>1$, что при любом $v \geqslant \hat{v}$ найдется вектор $\lambda \in \Lambda_{n}$, для которого верно равенство (17).

Действительно, на основании леммы 1 вектор-функция $\xi(u, v)$ с компонентами

$$
\xi_{i}\left(u_{i}, v\right)=v^{u_{i}}, \quad i \in N_{n},
$$

принадлежит классу $\Xi(f(X), 1, \hat{v})$, где $\hat{v}$ определяется согласно формуле (9). Поэтому следствие 5 легко получаем из следствия 4 . 
Следствие 6. Если $f(X) \subset \mathbf{R}_{+}^{n}$, то справедливы следующие утверждения.

При любом векторе $\lambda \in \Lambda_{n} u$ любом $v>0$ решение $x^{*} n$-KДЗ $(X, f)$, удовлетворяющее равенству

$$
\sum_{i=1}^{n} \lambda_{i} f_{i}^{v}\left(x^{*}\right)=\min \left\{\sum_{i=1}^{n} \lambda_{i} f_{i}^{v}(x): x \in X\right\},
$$

эффективно.

Если для $x^{*} \in P(X, f)$ выполняется включение $f\left(x^{*}\right) \in \mathbf{R}_{++}^{n}$, то существует такое число $\hat{v}>0$, что для любого $v \geqslant \hat{v}$ найдется вектор $\lambda \in \Lambda_{n}$, при котором верно равенство (18).

Утверждения этого следствия с очевидностью вытекают из совместного применения леммы 2 и следствия 4 для вектор-функции $\xi(u, v)$ с компонентами

$$
\xi_{i}\left(u_{i}, v\right)=u_{i}^{v}, \quad i \in N_{n} .
$$

Следствие 7. Пусть $f(X) \subset \mathbf{R}_{+}^{n}$ и справедлива формула

$$
\forall i \in N_{n} \quad \forall x, x^{\prime} \in X \quad\left(f_{i}(x)>f_{i}\left(x^{\prime}\right) \Rightarrow f_{i}(x) \geqslant n f_{i}\left(x^{\prime}\right)\right) .
$$

Тогда имеют место следующие утверждения.

При любом векторе $\lambda \in \Lambda_{n}$ решение $x^{*} n$-КДЗ $(X, f)$, удовлетворяющее равенству

$$
\sum_{i=1}^{n} \lambda_{i} f_{i}\left(x^{*}\right)=\min \left\{\sum_{i=1}^{n} \lambda_{i} f_{i}(x): x \in X\right\}
$$

эффективно.

Если $x^{*} \in P(X, f)$ u $f\left(x^{*}\right) \in \mathbf{R}_{++}^{n}$, то найдется вектор $\lambda \in \Lambda_{n}$, при котором верно равенство (19).

Доказательство. Легко видеть, что при выполнении условий следствия 7 векторфункция $\xi(u, v)$ с компонентами

$$
\xi_{i}\left(u_{i}, v\right)=u_{i} v, \quad i \in N_{n},
$$

принадлежит классу $\Xi(f(X), 0, \hat{v})$ при любом $\hat{v}>0$. Поэтому из первого утверждения следствия 4 вытекает первое утверждение.

Далее, так как $f\left(x^{*}\right) \in \mathbf{R}_{++}^{n}$, то при любых $v>0$ и $i \in N_{n}$ верно неравенство $\xi_{i}\left(f_{i}\left(x^{*}\right), v\right)>0$. Поэтому из второго утверждения следствия 4 находим, что при любом числе $v>0$ существует вектор $\lambda \in \Lambda_{n}$, для которого выполняется равенство

$$
\sum_{i=1}^{n} \lambda_{i} v f_{i}\left(x^{*}\right)=\min \left\{\sum_{i=1}^{n} \lambda_{i} v f_{i}(x): x \in X\right\} .
$$

Отсюда, учитывая неравенство $v>0$, получаем, что

$$
\sum_{i=1}^{n} \lambda_{i} f_{i}\left(x^{*}\right)=\min \left\{\sum_{i=1}^{n} \lambda_{i} v f_{i}(x): x \in X\right\} .
$$

Следствие 7 доказано. 
Заметим, что в следствиях 5-7 для всякого эффективного решения $x^{*}$ в качестве вектора $\lambda$ можно взять вектор, компоненты которого задаются формулой (15).

Наконец, сформулируем следствие, являющееся аналогом теоремы 2 из статьи [4].

Следствие 8. Если решение $x^{*} n-K Д З ~(X, f)$ и возрастающие функции

$$
\eta_{i}: f_{i}(X) \rightarrow \mathbf{R}_{+}, \quad i \in N_{n}
$$

подчинены условиям

$$
\eta_{1}\left(f_{1}\left(x^{*}\right)\right)=\eta_{2}\left(f_{2}\left(x^{*}\right)\right)=\ldots=\eta_{n}\left(f_{n}\left(x^{*}\right)\right),
$$

то справедливы следующие утверждения.

При любом $v>0$ решение $x^{*} \in X$, удовлетворяющее равенству

$$
n \eta_{1}^{v}\left(f_{1}\left(x^{*}\right)\right)=\min \left\{\sum_{i=1}^{n} \eta_{i}^{v}\left(f_{i}\left(x^{*}\right)\right): x \in X\right\}
$$

эффективно.

Eсли $x^{*} \in P(X, f)$, то существует такое $\hat{v}>0$, что для любого $v \geqslant \hat{v}$ справедливо равенство (21).

Доказательство. На основании леммы 5 вектор-функция $\xi(u, v)$ с компонентами

$$
\xi_{i}\left(u_{i}, v\right)=\eta_{i}^{v}\left(u_{i}\right), \quad i \in N_{n}
$$

принадлежит классу $\Xi(f(X), 0, \hat{v})$, где число $\hat{v}$ задается формулой (11). Поэтому из теоремы 1 , учитывая равенство (20), выводим первое утверждение.

Далее, применяя теорему 2 и вновь используя равенства (20), получаем второе утверждение следствия 8.

\section{Список литературы}

1. Подиновский В. В., Ногин В. Д., Парето-оптималъные решения многохритериалъных задач. Наука, Москва, 1982.

2. Дубов Ю. А., Травкин С. И., Якимец В. Н., Многохритериалъные модели формирования и выбора вариантов систем. Наука, Москва, 1986.

3. Емеличев В. А., Янушкевич О. А., Условия Парето-оптимальности в дискретных векторных задачах оптимизации. Дискретная математиха (1997) 9, Nㅗ, 154-160.

4. Емеличев В. А., Пашкевич А. В., Янушкевич О. А., Условия эффективности решения векторной задачи дискретной оптимизации. Дискретная математика (1999) 11, №1, $140-145$.

Статья поступила 02.05.2001. 